\title{
Optimal Motion Strategies for Range-Only Distributed Target Tracking
}

\author{
Ke X. Zhou and Stergios I. Roumeliotis
}

\begin{abstract}
In this paper we study the problem of optimal trajectory generation for a team of mobile robots that tracks a moving target using range-only measurements. We propose an adaptive-relaxation algorithm for determining the set of feasible locations that each robot must move to in order to collect the most informative measurements; i.e., distance measurements that minimize the uncertainty about the position of the target. We prove that the motion strategy that minimizes the trace of the position error covariance matrix is equivalent to the one that minimizes its maximum eigenvalue. The proposed method is applicable regardless of the process model employed for describing the motion of the target while its computational complexity is linear in the number of robots. Extensive simulation results are presented, demonstrating that the performance attained with the proposed method is comparable to that obtained with exhaustive search whose computational cost is exponential in the number of robots.
\end{abstract}

\section{INTRODUCTION}

Target tracking has recently attracted significant interest in the research community, because of its importance in a variety of applications, such as surveillance [1], defence applications [2], etc. In order to obtain increased tracking accuracy and to be able to monitor a sizeable area, a large number of sensors is often utilized for tracking. When multiple nodes obtain measurements of a target of interest while communicating over a wireless network, the acquired data can be processed either at a central fusion center, or in a distributed fashion, so as to estimate the target's trajectory [3].

As an alternative to using static sensors, the deployment of mobile sensors (i.e., robots) for tracking, offers significant advantages. By providing mobility to the sensors, a larger area can be covered without the need to increase the number of nodes in the sensing network. Additionally, the spatial distribution of the sensors can be changed dynamically in order to adapt to the motion of the target. For example, a team of robots can actively pursue a target and avert the target's evasion from the sensors' visibility range [4].

Regardless of the details of the implementation that is employed in a given application, the processing of every new measurement by a networked tracking system incurs a penalty in terms of use of communication bandwidth and CPU time, as well as in terms of power consumption. Since these resources are inevitably limited, it is necessary to devise algorithms that guarantee their optimal utilization [5]. Moreover, in many tracking applications the time needed for determining the

This work was supported by NASA (Grant No. 1263201) and NSF (ITR0324864, MRI-0420836)

Ke X. Zhou is with the Dept. of Electrical and Computer Engineering, Univ. of Minnesota, MN 55455, kezhouecs . umn.edu

Stergios I. Roumeliotis is with the Dept. of Computer Science and Engineering, Univ. of Minnesota, MN 55455, stergios@cs . umn.edu trajectory of a target is of critical importance (e.g., when tracking a hostile target). In such scenarios, the need for optimal tracking performance is even more compelling, and provides the motivation for our work.

In this paper, we study the problem of determining optimal trajectories for a team of robots that track a moving target using range-only measurements. Since the measurement model is non-linear, the locations where distance measurements are collected have a profound effect on the accuracy of this estimation process. Optimality in this case is sought with respect to the accuracy of the target's localization accuracy, i.e., we seek to minimize the trace of the covariance matrix of the target's position estimates. It it shown, that regardless of the target's motion model, this optimization problem can be exactly reformulated as that of minimizing the norm of the sum of a set of vectors of known length. The direction of motion of each robot affects the direction of the corresponding vector, while the speed of motion determines the constraint on the angular range of each vector.

A novel adaptive relaxation algorithm is proposed for solving this problem, and it is shown through extensive simulation studies, that the performance attainable with the proposed method is comparable to that obtained with an exhaustive search-based algorithm. While the computational complexity of exhaustive search is prohibitively large (exponential in the number of robots), the proposed adaptive relaxation method has complexity linear in the number of robots, and is thus well-suited for real-time implementations.

\section{Literature REVIEW}

Target tracking has received considerable attention in the literature (e.g., [6], [7]). In most cases, however, the sensors involved are static and the emphasis is on the optimal processing of the available information rather than the placement or repositioning of the sensors. The idea of choosing sensing locations in order to maximize information gain is also known as adaptive sensing or active perception [8]. In what follows, we present a few representative examples of related work in optimal trajectory generation for parameter detection, Simultaneous Localization And Mapping (SLAM), and tracking in particular.

In [9] the authors investigate motion strategies for a team of robots to gather the most informative concentration measurements necessary for determining the parameters of a pointsource gas release. To this end, they employ a nonlinear least squares estimator, and perform an approximate one-step-ahead minimization of the trace of the expected covariance matrix in order to determine a locally optimal robot motion. The robot velocity is considered fixed and the robot heading is used as 
the optimization variable. The heading directions of the robots are not optimized simultaneously but sequentially. Each robot accounts only for the expected information gain by the robots that have already determined their next movement.

Feder et al. [8] propose an adaptive sensing strategy for SLAM, that determines the next best action by numerically minimizing a cost function determined by the volume of the error ellipses of landmark positions and robot pose. In this scenario, the authors consider the case of robot localization using static features. The authors comment on the intractability and poor scaling of analytical minimization.

An approach, where trajectories of robot teams are optimized in order to maximize information gain during cooperative localization, is presented by Trawny and Barfoot in [10]. In a sense, this problem also corresponds to optimal tracking, but in this case of the own teammates. Contrary to regular target tracking, here the trajectories of both tracker and tracked object are controllable. The algorithm works by choosing optimal trajectory parameters that minimize the determinant of the covariance matrix using a Sequential Quadratic Programming technique. This approach scales poorly in the number of team members or the number of trajectory parameters.

The work that is closely related to the problem addressed in this paper is that of Stroupe and Balch [11]. In [11], the authors propose an approximately optimal tracking behavior, in which the robots attempt to minimize the target location uncertainty. The objective function is the determinant of the target location covariance matrix. The authors do not optimize over all sets of possible robot trajectories, but instead optimize each robot's next manoeuver separately. Additionally, they incorporate expected knowledge from the teammates' actions by approximating them through a measurement from the other robots' current locations. The algorithm then performs a greedy search over a discretized set of possible robot headings, separately for each robot.

One of the main drawbacks of the approach in [11] is that the minimization criterion does not appropriately represent the uncertainty of the target. If for example the target-position covariance matrix is close to becoming singular, its determinant will be approximately zero regardless of the values of the individual components of the diagonal. Furthermore, the location of the target is estimated using a Kalman filter which is the optimal estimator that minimizes the trace of the covariance matrix. It is therefore appropriate to employ as objective function for determining the sensors' trajectories the same one employed for deriving the estimator used. Additionally, the algorithm in [11] only approximately accounts for the fellow robots' contributions, thus allowing it to scale linearly in the number of robots. Our algorithm, based on adaptive relaxation, has complexity linear in the number of robots and performs simultaneous optimization of every robot's heading.

\section{PROBLEM Formulation}

Consider a group of mobile robots moving in a plane and tracking the position of a moving target by processing distance measurements. In this paper, we study the case of global tracking, i.e., the position of the target is determined with respect to a fixed (global) frame of reference, instead of a relative group-centered one. We hereafter employ the assumption that the position and orientation of each of the robots is known with high accuracy within the global frame of reference (this is the case, for example, when all robots have access to precise GPS and compass measurements). As a result, we are able to ignore the uncertainty in the robots' pose when formulating the tracking equations.

\section{A. State Propagation}

In this work, the Extended Kalman Filter (EKF) is used for recursively estimating the target's state, $X_{T}(k)$. This is defined as a vector of dimension $2 N$, and can include components such as position, velocity, and acceleration:

$$
X_{T}(k)=\left[x_{T}(k) y_{T}(k) \dot{x}_{T}(k) \dot{y}_{T}(k) \ddot{x}_{T}(k) \ddot{y}_{T}(k) \ldots\right]^{\mathrm{T}}
$$

The discrete-time state propagation equation is:

$$
X_{T}(k+1)=\Phi_{k} X_{T}(k)+G_{k} \mathbf{w}_{d}(k)
$$

where $\mathbf{w}_{d}$ is a zero-mean white Gaussian noise process with variance $Q_{d}=E\left[\mathbf{w}_{d}(k) \mathbf{w}_{d}^{\mathrm{T}}(k)\right]$.

The estimate of the target's state is propagated by:

$$
\hat{X}_{T}(k+1 \mid k)=\Phi_{k} \hat{X}_{T}(k \mid k)
$$

where $\hat{X}_{T}(\ell \mid j)$ is the estimate for the target state at time step $\ell$, after measurements up to time step $j$ have been processed. The error-state covariance matrix is propagated as:

$$
P_{k+1 \mid k}=\Phi_{k} P_{k \mid k} \Phi_{k}^{\mathrm{T}}+G_{k} Q_{d} G_{k}^{\mathrm{T}}
$$

where $P_{\ell \mid j}$ is the covariance of the target state estimate $\hat{X}_{T}(\ell \mid j)$. The state transition matrix, $\Phi_{k}$, and the process noise Jacobian, $G_{k}$, that appear in the preceding expressions depend on the motion model used [7]. In our approach, these can be arbitrary matrices, since no assumptions on their properties are imposed.

\section{B. Measurement model}

At time-step $k+1$, each robot of the team measures its distance to the target, and therefore the measurement equation is:

$$
\begin{aligned}
Z(k+1) & =\left[d_{1}(k+1) \ldots d_{M}(k+1)\right]^{\mathrm{T}}+\left[n_{1}(k+1) \ldots n_{M}(k+1)\right]^{\mathrm{T}} \\
& =\mathbf{d}(k+1)+\mathbf{n}(k+1)
\end{aligned}
$$

where

$d_{i}(k+1)=\sqrt{\left(x_{T}(k+1)-x_{i}(k+1)\right)^{2}+\left(y_{T}(k+1)-y_{i}(k+1)\right)^{2}}$

for $i=1, \ldots, M$, where $M$ is the number of robots, $\left[x_{i}(k+\right.$ 1) $\left.y_{i}(k+1)\right]^{\mathrm{T}}$ is the position of robot $i$ at time $k+1$, and $n_{i}(k+1)$ is the noise in the $i$-th robot's distance measurement, which is a zero-mean white Gaussian process with variance $E\left[n_{i}^{2}(k+1)\right]=\sigma_{i}^{2}$. We consider the case where the noise in the distance measurements of different robots is uncorrelated, i.e, $E\left[n_{i}(k+1) n_{j}(k+1)\right]=0$.

It is clear that the measurement equation (Eq. (3)) is a nonlinear function of the state variables. The measurement error equation, obtained by linearizing Eq. (3) is:

$$
\tilde{Z}(k+1) \simeq H_{k+1} \tilde{X}_{T}(k+1)+\mathbf{n}(k+1)
$$


where

$$
\begin{aligned}
\tilde{Z}(k+1) & =Z(k+1)-\hat{Z}(k+1 \mid k) \\
\tilde{X}_{T}(k+1) & =X_{T}(k+1)-\hat{X}_{T}(k+1 \mid k) \\
H_{k+1} & =\left[\begin{array}{ll}
H_{e, k+1} & \mathbf{0}_{M \times(2 N-2)}
\end{array}\right] \\
H_{e, k+1}^{\mathrm{T}} & =\left[\begin{array}{lll}
\cos \theta_{1}(k+1) & \ldots & \cos \theta_{M}(k+1) \\
\sin \theta_{1}(k+1) & \ldots & \sin \theta_{M}(k+1)
\end{array}\right] \\
\cos \theta_{i}(k+1) & =\frac{\hat{x}_{T}(k+1 \mid k)-x_{i}(k+1)}{\hat{d}_{i}(k+1)} \\
\sin \theta_{i}(k+1) & =\frac{\hat{y}_{T}(k+1 \mid k)-y_{i}(k+1)}{\hat{d}_{i}(k+1)} \\
\hat{d}_{i}(k+1) & =\sqrt{\widehat{\Delta x_{T i}^{2}}(k+1)+\widehat{\Delta y}_{T i}^{2}(k+1)} \\
\widehat{\Delta x}_{T i}(k+1) & =\hat{x}_{T}(k+1 \mid k)-x_{i}(k+1) \\
\widehat{\Delta y}_{T i}(k+1) & =\hat{y}_{T}(k+1 \mid k)-y_{i}(k+1)
\end{aligned}
$$

The angle $\theta_{i}$ that appears in the preceding equations represents the bearing angle of robot $i$ towards the target, expressed in global coordinates (cf. Fig. 1).

The covariance of the target state estimate is updated as:

$$
P_{k+1 \mid k+1}^{-1}=P_{k+1 \mid k}^{-1}+H_{k+1}^{\mathrm{T}} R^{-1} H_{k+1}
$$

where $R=\operatorname{diag}\left(\sigma_{i}^{2}\right)$ is the covariance matrix of all the measurements performed at time step $k$.

At this point we define the following partitioning for the state covariance matrix:

$$
P_{\ell \mid j}=\left[\begin{array}{ll}
P_{\ell \mid j, 11} & P_{\ell \mid j, 12} \\
P_{\ell \mid j, 12}^{\mathrm{T}} & P_{\ell \mid j, 22}
\end{array}\right]
$$

where $P_{\ell \mid j, 11}$ denotes the covariance for the position, $\left[x_{T} y_{T}\right]^{\mathrm{T}}$, of the target at time step $\ell$ given measurements up to time step $j$, and for the inverse state covariance matrix $P_{k+1 \mid k}$ :

$$
P_{k+1 \mid k}^{-1}=\left[\begin{array}{ll}
A_{11} & A_{12} \\
A_{12}^{\mathrm{T}} & A_{22}
\end{array}\right]
$$

Moreover, we observe that

$H_{k+1}^{\mathrm{T}} R^{-1} H_{k+1}=\left[\begin{array}{cc}H_{e, k+1}^{\mathrm{T}} R^{-1} H_{e, k+1} & \mathbf{0}_{2 \times(N-2)} \\ \mathbf{0}_{(N-2) \times 2} & \mathbf{0}_{(N-2) \times(N-2)}\end{array}\right]$

and thus, Eq. (9) yields:

$$
P_{k+1 \mid k+1}=\left[\begin{array}{cc}
A_{11}+H_{e, k+1}^{T} R^{-1} H_{e, k+1} & A_{12} \\
A_{12}^{T} & A_{22}
\end{array}\right]^{-1}
$$

In this work, we are interested in the accuracy of the position estimates for the target, and therefore we now seek an expression for the covariance matrix, $P_{k+1 \mid k+1,11}$, of the target's position estimates after the update. By employing the properties of the Schur complement for the inversion of a partitioned matrix, in Eq. (12), we obtain

$$
\begin{aligned}
P_{k+1 \mid k+1,11} & =\left(A_{11}+H_{e, k+1}^{\mathrm{T}} R^{-1} H_{e, k+1}-A_{12} A_{22}^{-1} A_{12}^{\mathrm{T}}\right)^{-1} \\
& =\left(P_{k+1 \mid k, 11}^{-1}+H_{e, k+1}^{\mathrm{T}} R^{-1} H_{e, k+1}\right)^{-1}
\end{aligned}
$$

where in the second equality we have used the Schur complement for the inversion of the partitioned matrix $P_{k+1 \mid k}$ (cf. Eq. (11)).

\section{Problem Statement and Reformulation}

From Eqs. (6)-(8) and (13) it is apparent that the covariance matrix of the target's position estimates after each update step will depend on all the new positions, $p_{i}(k+1)=\left[x_{i}(k+\right.$ 1) $\left.y_{i}(k+1)\right]^{\mathrm{T}}, i=1 \ldots M$, where each of the robots will move to so as to record its next distance measurements $d_{i}(k+1)$. Therefore, we seek to determine the optimal motion strategy for all robots, in order to attain the best possible estimation performance. Assuming that at time-step $k$, the $i$ th robot is at location $p_{i}(k)=\left[\begin{array}{ll}x_{i}(k) & y_{i}(k)\end{array}\right]^{\mathrm{T}}$, and that it moves with velocity $v_{i}(k)$, at time step $k+1$, its position will be:

$$
\begin{array}{r}
x_{i}(k+1)=x_{i}(k)+v_{i}(k) \delta t \cos \varphi_{i}(k) \\
y_{i}(k+1)=y_{i}(k)+v_{i}(k) \delta t \sin \varphi_{i}(k)
\end{array}
$$

where $\varphi_{i}(k) \in[0,2 \pi)$ is the heading direction of the robot.

We thus see that given the current position, $p_{i}(k)$, and velocity, $v_{i}(k)$, of the robots, their positions at the next timestep, $p_{i}(k+1)$, and hence the covariance for the target's position after the update (cf. Eq. (13)) are functions of the robots' current motion directions $\varphi_{i}(k)$. The problem we address in this work is that of determining the set of heading directions of the robots that minimizes the trace of the target's position estimates, i.e.,

$$
\left\{\varphi_{1}(k), \ldots, \varphi_{M}(k)\right\}=\underset{\varphi_{1}(k), \ldots, \varphi_{M}(k)}{\arg \min } \operatorname{tr}\left(P_{k+1 \mid k+1,11}\right)
$$

From Eqs. (7), (8), (14) and (15), as well as from Fig. 1, we conclude that the heading angle of each robot, $\varphi_{i}(k)$, and the bearing angle towards the target, $\theta_{i}(k+1)$, are closely related. In particular, if $\theta_{i}(k+1)$ is known then $\varphi_{i}(k)$ is determined by

$$
\begin{aligned}
& \varphi_{i}(k)=\theta_{i}(k+1)+\arcsin \left(\xi_{i}(k+1)\right) \quad \text { or } \\
& \varphi_{i}(k)=\theta_{i}(k+1)+\pi-\arcsin \left(\xi_{i}(k+1)\right)
\end{aligned}
$$

where

$$
\begin{aligned}
\xi_{i}(k+1)= & \frac{\left(\hat{y}_{T}(k+1 \mid k)-y_{i}(k)\right) \cos \theta_{i}(k+1)}{v_{i}(k) \delta t} \\
& -\frac{\left(\hat{x}_{T}(k+1 \mid k)-x_{i}(k)\right) \sin \theta_{i}(k+1)}{v_{i}(k) \delta t}
\end{aligned}
$$

From the two equivalent solutions in Eq. (16), the robot $i$ will choose the one that brings it closer to the target so as to increase the probability of re-detection later on.

Since each $\varphi_{i}(k), i=1 \ldots M$ is a function of $\theta_{i}(k+1)$, $i=1 \ldots M$, we can reformulate the optimization problem as the search for the optimal set of $\theta_{i}(k+1)$

$$
\left\{\theta_{1}, \ldots, \theta_{M}\right\}=\underset{\theta_{1}(k+1), \ldots, \theta_{M}(k+1)}{\arg \min } \operatorname{tr}\left(P_{k+1 \mid k+1,11}\right)
$$

Once $\left\{\theta_{1}(k+1), \ldots, \theta_{M}(k+1)\right\}$ are determined, the optimal set of heading directions $\left\{\varphi_{1}(k), \ldots, \varphi_{M}(k)\right\}$ can be evaluated using Eq. (16).

At this point, we should note that although the variables $\left\{\varphi_{1}(k), \ldots, \varphi_{M}(k)\right\}$ are unconstrained, the bearing angles, $\left\{\theta_{1}(k+1), \ldots, \theta_{M}(k+1)\right\}$, are constrained by the fact that the velocity, $v_{i}(k)$, of each robot, is bounded by some constant $v_{\text {imax }}$. As evident from Fig. 1, at the next time step, a given 


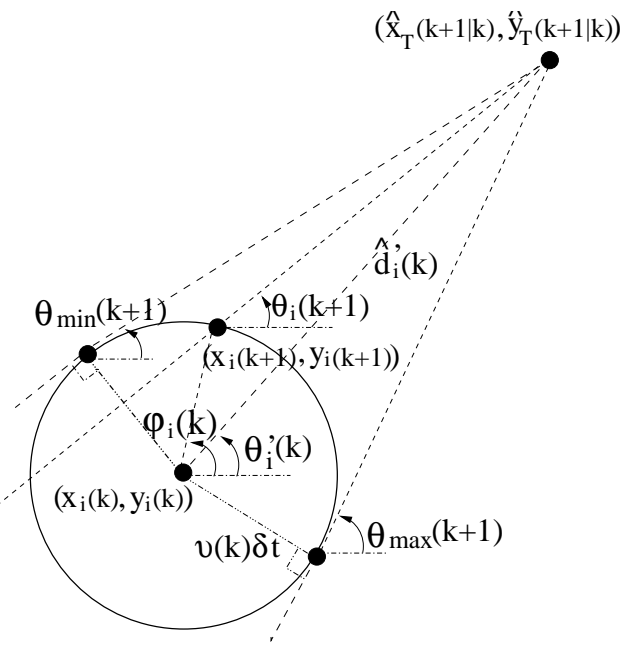

Fig. 1. Bearing Angle Constraints.

robot can only move within a circle centered at its current position and with radius $v_{\text {imax }} \delta t$. Therefore, for $\hat{d}_{i}^{\prime}(k) \geq$ $v_{\text {imax }} \delta t, \theta_{i}(k+1)$ can only change within a given range:

$$
\left[\theta_{\text {imin }}(k+1), \theta_{\text {imax }}(k+1)\right]=\left[\theta_{i}^{\prime}(k)-\eta_{k}, \theta_{i}^{\prime}(k)+\eta_{k}\right]
$$

where $\eta_{k}=\arcsin \left(\frac{v_{i m a x} \delta t}{\hat{d}_{i}^{\prime}(k)}\right)$ and

$$
\begin{aligned}
& \hat{d}_{i}^{\prime}(k)=\sqrt{\left(\hat{x}_{T}(k+1 \mid k)-x_{i}(k)\right)^{2}+\left(\hat{y}_{T}(k+1 \mid k)-y_{i}(k)\right)^{2}} \\
& \theta_{i}^{\prime}(k)=\operatorname{Atan} 2\left(\hat{y}_{T}(k+1 \mid k)-y_{i}(k), \hat{x}_{T}(k+1 \mid k)-x_{i}(k)\right)
\end{aligned}
$$

are the distance and bearing angle from the current location of the $i$-th robot to that of the next (estimated) target position, respectively. If, however, $\hat{d}_{i}^{\prime}(k)<v_{i \max } \delta t$, the $\theta_{i}(k+1) \in$ $[0,2 \pi)$.

Notice that at each time step $P_{k+1 \mid k, 11}$ is fixed and the only variables affecting the trace of the error-state covariance, $P_{k+1 \mid k+1,11}$, are the $\theta_{i}(k+1)$ 's (cf. Eqs. (13), (6)). If the maximum speed of the robots is increased, the range of values for the variables $\theta_{i}(k+1)$ will increase (relaxation of the constraints). Thus, in most cases, the optimal trace of the position covariance matrix will decrease.

We now derive a closed-form expression for the objective function, as a function of the variables $\theta_{i}(k+1)$. Note that since the matrix $P_{k+1 \mid k}$ is positive definite, so is $P_{k+1 \mid k, 11}$. The singular value decomposition of $P_{k+1 \mid k, 11}^{-1}$ is:

$$
P_{k+1 \mid k, 11}^{-1}=U \Sigma U^{\mathrm{T}}
$$

where

$$
U=\left[\begin{array}{cc}
\cos \theta_{0} & -\sin \theta_{0} \\
\sin \theta_{0} & \cos \theta_{0}
\end{array}\right], \Sigma=\operatorname{diag}\left(\eta_{1}^{2}, \eta_{2}^{2}\right), \eta_{1}^{2} \geq \eta_{2}^{2}
$$

Substituting Eq. (19) into the right-hand side of Eq. (13), and noting that $U U^{\mathrm{T}}=U^{\mathrm{T}} U=I_{2 \times 2}$ and that similarity transformations do not change the trace of a matrix, we obtain:

$$
\operatorname{tr}\left(P_{k+1 \mid k+1,11}\right)=\operatorname{tr}\left(\left(\Sigma+H_{n, k+1}^{\mathrm{T}} R^{-1} H_{n, k+1}\right)^{-1}\right)
$$

where $H_{n, k+1}=H_{e, k+1} U$, and

$$
H_{n, k+1}=\left[\begin{array}{ccc}
\cos \tilde{\theta}_{1}(k+1) & \ldots & \cos \tilde{\theta}_{M}(k+1) \\
\sin \tilde{\theta}_{1}(k+1) & \ldots & \sin \tilde{\theta}_{M}(k+1)
\end{array}\right]^{\mathrm{T}}
$$

with $\tilde{\theta}_{i}(k+1)=\theta_{i}(k+1)-\theta_{0}, i=1, \ldots, M$.

We can then write matrix $\Sigma+H_{n, k+1}^{\mathrm{T}} R^{-1} H_{n, k+1}$ in Eq. (20) as:

$$
\left[\begin{array}{cc}
\eta_{1}^{2}+\sum_{i=1}^{M} \sigma_{i}^{-2} \cos ^{2} \tilde{\theta}_{i} & \sum_{i=1}^{M} \sigma_{i}^{-2} \cos \tilde{\theta}_{i} \sin \tilde{\theta}_{i} \\
\sum_{i=1}^{M} \sigma_{i}^{-2} \cos \tilde{\theta}_{i} \sin \tilde{\theta}_{i} & \eta_{2}^{2}+\sum_{i=1}^{M} \sigma_{i}^{-2} \sin ^{2} \tilde{\theta}_{i}
\end{array}\right]
$$

where for simplicity we have dropped the time indices from $\tilde{\theta}_{i}(k+1)$, and therefore its inverse is:

$$
\begin{aligned}
& \left(\Sigma+H_{n, k+1}^{\mathrm{T}} R^{-1} H_{n, k+1}\right)^{-1}= \\
& \frac{1}{\Delta}\left[\begin{array}{cc}
\eta_{2}^{2}+\sum_{i=1}^{M} \sigma_{i}^{-2} \sin ^{2} \tilde{\theta}_{i} & -\sum_{i=1}^{M} \sigma_{i}^{-2} \cos \tilde{\theta}_{i} \sin \tilde{\theta}_{i} \\
-\sum_{i=1}^{M} \sigma_{i}^{-2} \cos \tilde{\theta}_{i} \sin \tilde{\theta}_{i} & \eta_{1}^{2}+\sum_{i=1}^{M} \sigma_{i}^{-2} \cos ^{2} \tilde{\theta}_{i}
\end{array}\right]
\end{aligned}
$$

where $\Delta=\operatorname{det}\left(\Sigma+H_{n, k+1}^{\mathrm{T}} R^{-1} H_{n, k+1}\right)$.

Substituting Eq. (21) in Eq. (20), we have:

$$
\operatorname{tr}\left(P_{k+1 \mid k+1,11}\right)=\frac{1}{\Delta}\left(\eta_{1}^{2}+\eta_{2}^{2}+\sum_{i=1}^{M} \sigma_{i}^{-2}\right)
$$

Noting that the numerator is constant, we have the equivalence:

$$
\min \operatorname{tr}\left(P_{k+1 \mid k+1,11}\right) \Leftrightarrow \max \Delta=\operatorname{det}\left(P_{k+1 \mid k+1,11}^{-1}\right)
$$

By expanding the expression of the determinant $\Delta$, it can be shown [12] that maximizing $\Delta$ is equivalent to minimizing:

$$
\Delta^{\prime}=\left\|\lambda_{0}+\sum_{i=1}^{M} \lambda_{i} \exp \left(j 2 \tilde{\theta}_{i}(k+1)\right)\right\|_{2}
$$

where $\lambda_{0}=\eta_{1}^{2}-\eta_{2}^{2} \geq 0, \lambda_{i}=\sigma_{i}^{-2} \geq 0, j=\sqrt{-1}$.

We thus see that the original problem of minimizing the trace of the covariance matrix of the target's position estimates is exactly reformulated to the following minimization problem:

$$
\min \left\|\lambda_{0}+\sum_{i=1}^{M} \lambda_{i} \exp \left(j 2 \tilde{\theta}_{i}(k+1)\right)\right\|_{2}
$$

under the constraints:

$\tilde{\theta}_{\text {imin }}(k+1) \leq \tilde{\theta}_{i}(k+1) \leq \tilde{\theta}_{\text {imax }}(k+1), i=1, \ldots, M$

In this last expression, $\tilde{\theta}_{i m i n}(k+1)=\theta_{\text {imin }}(k+1)-\theta_{0}$ and $\tilde{\theta}_{i \max }(k+1)=\theta_{\text {imax }}(k+1)-\theta_{0}$ (cf. Eq. (18)).

From Eq. (25) we observe that the original problem has been transformed into the minimization of the length of the sum of $M+1$ vectors in $2-D$. The vector $V_{0}=\left[\begin{array}{ll}\lambda_{0} & 0\end{array}\right]^{\mathrm{T}}$ remains constant, while each of the vectors $V_{i}=\lambda_{i} \exp \left(j 2 \tilde{\theta}_{i}(k+1)\right)$, has fixed length, $\lambda_{i}$, while its orientation varies under the constraints in Eq. (26).

We note at this point, that the original minimization criterion was the trace of the covariance matrix of the target's position estimates. Another meaningful optimization criterion is the minimization of the maximum eigenvalue of the covariance matrix. Interestingly, it can be shown [12], that for this particular problem the two criteria are equivalent, i.e., they result in the same solution. Additionally, as described in [12], the simple and intuitive case of one sensor $(M=1)$, is equivalent to the minimization of a Rayleigh Quotient. 


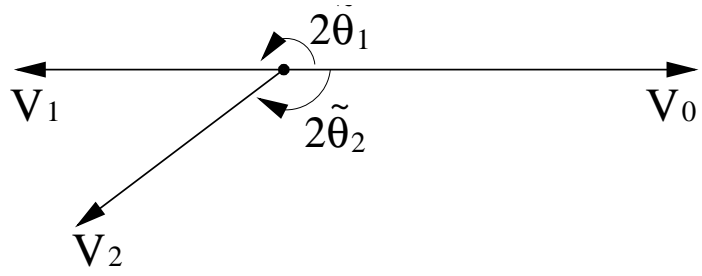

Fig. 2. Illustration of Standard Relaxation

\section{Computing the Solution}

If the number of robots, $M$, is larger than one, in general the problem (25) is a non-convex optimization problem [12], and thus gradient-based methods need to be used with caution, since these methods are susceptible to the existence of local minima.

1) Standard Relaxation Method: A relaxation method is often applied when addressing an optimization problem which is non-convex. Such methods have low computational complexity, and are easy to implement. However, they often return locally optimal points and can not ensure convergence to a globally optimal solution.

For the optimization problem Eq. (25), a relaxation algorithm calculates the next optimal point $\tilde{\theta}_{i_{N E W}}(k+1)$ in each iteration as:

$$
\underset{\tilde{\theta}_{i}}{\arg \min }\left\|\lambda_{0}+\sum_{\kappa=1, \kappa \neq i}^{M} \lambda_{\kappa} \exp \left(j 2 \tilde{\theta}_{\kappa_{O L D}}\right)+\lambda_{i} \exp \left(j 2 \tilde{\theta}_{i}\right)\right\|_{2}
$$

under the constraints $\tilde{\theta}_{\text {imin }}(k+1) \leq \tilde{\theta}_{i} \leq \tilde{\theta}_{\text {imax }}(k+1)$, where $\theta_{\kappa_{O L D}}$ are the vector directions computed, sequentially, in the previous iteration of the algorithm.

It is easy to demonstrate that such an approach is prone to being trapped in local minima. For example, consider the simple case where $M=2$ and no constraints are imposed on $\tilde{\theta}_{i}(k+1), i=1,2$. We assume the initial values $2 \tilde{\theta}_{1}=\pi$, $2 \tilde{\theta}_{2}=-\frac{5 \pi}{6}$, and $\lambda_{0}=\sqrt{2}, \lambda_{1}=\lambda_{2}=1$, as shown in Fig. 2 .

The results of applying relaxation in this case are:

$$
\begin{aligned}
\text { Initial Condition : } 2 \tilde{\theta}_{2} & =-2.6180,2 \tilde{\theta}_{1}=3.1416 \\
\text { 1st Iteration : } 2 \tilde{\theta}_{2} & =3.1416,2 \tilde{\theta}_{1}=-3.1416 \\
\text { 2nd Iteration }: 2 \tilde{\theta}_{2} & =-3.1416,2 \tilde{\theta}_{1}=3.1416 \\
\text { 3rd Iteration }: 2 \tilde{\theta}_{2} & =-3.1416,2 \tilde{\theta}_{1}=3.1416
\end{aligned}
$$

So the objective value that standard relaxation returns in this case is $(2-\sqrt{2})$, while the true global minimum of the objective function is 0 ; this is obtained when $2 \tilde{\theta}_{1}=-2 \tilde{\theta}_{2}=$ $3 \pi / 4$.

2) Grid Search Method: Ideally, a grid search method should return the global optimal solution if the grid size is small enough. However, in practice this is hard to guarantee since the computational complexity and storage needs are exponential in the number of robots, and the practical restrictions become prohibitive when the number of robots, $M$, is large. We here use the grid search method as a benchmark to compare its results with these of our algorithm in the simulations of Section IV.

3) Adaptive Relaxation Method: We now propose an adaptive relaxation method to solve the problem in Eq. (25). This algorithm adopts the basic idea from standard relaxation, but with some changes. In the standard relaxation algorithm, we determine the solution $\tilde{\theta}_{i}(k+1)$, while keeping all the $\tilde{\theta}_{\kappa}(k+1)$ $(\kappa \neq i)$ fixed. Because the standard relaxation gets easily trapped at local minima, at every iteration we now introduce a perturbation vector, proportional to the sum of the vectors of the previous iteration. Thus, at every iteration, $\tilde{\theta}_{i_{N E W}}(k+1)$ is obtained by minimizing $\left(\arg \min _{\tilde{\theta}_{i}}\right)$ the quantity:

$$
\left\|\lambda_{0}+\sum_{\kappa=1, \kappa \neq i}^{M} \lambda_{\kappa} \exp \left(j 2 \tilde{\theta}_{\kappa_{O L D}}\right)+\lambda_{i} \exp \left(j 2 \tilde{\theta}_{i}\right)+V_{M+1}\right\|_{2}
$$

where

$$
V_{M+1}=-\alpha\left(\lambda_{0}+\sum_{\kappa=1}^{M} \lambda_{\kappa} \exp \left(j 2 \tilde{\theta}_{\kappa_{O L D}}\right)\right)
$$

under the constraint $\tilde{\theta}_{\text {imin }}(k+1) \leq \tilde{\theta}_{i}(k+1) \leq \tilde{\theta}_{\text {imax }}(k+$ $1)$. The parameter $\alpha \in[0,1]$ is termed the relaxation factor. When $\alpha=0$ the method becomes identical to standard relaxation, while at the other extreme, when $\alpha=1, \tilde{\theta}_{i_{N E W}}(k+$ $1)=\tilde{\theta}_{i_{O L D}}(k+1)$, and therefore the solution does not change between iterations. We thus see that in the adaptive relaxation method, we introduce a perturbation that "slows down" the convergence rate of the estimates, by smoothing the cost function. This makes the algorithm less sensitive to local minima. For the example in Fig. 2, by employing the adaptive relaxation the results are:

$$
\begin{array}{r}
\text { Initial Condition : } 2 \tilde{\theta}_{2}=-2.6180,2 \tilde{\theta}_{1}=3.1416 \\
\text { 1st Iteration : } 2 \tilde{\theta}_{2}=-2.7693,2 \tilde{\theta}_{1}=2.9824 \\
\text { 2nd Iteration : } 2 \tilde{\theta}_{2}=-2.7745,2 \tilde{\theta}_{1}=2.8053 \\
\ldots \\
\text { 16th Iteration : } 2 \tilde{\theta}_{2}=-2.3562,2 \tilde{\theta}_{1}=2.3562 \\
\text { 17th Iteration : } 2 \tilde{\theta}_{2}=-2.3562,2 \tilde{\theta}_{1}=2.3562
\end{array}
$$

Noting that $2.3562=3 \pi / 4$, we see that the adaptive relaxation method returns the global minimum point, in this case.

For clarity, we present the adaptive relaxation algorithm in a more concrete form below:

1) Calculate $V_{M+1}$ through Eq. (29).

2) for $i=1: M$

Determine $\tilde{\theta}_{i_{N E W}}(k+1)$ through Eq. (28).

end

3) Update all $\tilde{\theta}_{i}(k+1)$, i.e., assign $\tilde{\theta}_{i_{O L D}}(k+1)=$ $\tilde{\theta}_{i_{N E W}}(k+1)$, for $i=1: M$.

4) Return to step 1 until the maximum number of iterations is reached.

The adaptive relaxation process has similar computational complexity to the standard relaxation algorithm, it is easily implemented and its memory requirements are low.

\section{Simulation Results}

The simulations shown here are implemented in MATLAB. We plot the trace of the position covariance matrix for robot motions computed by employing the adaptive relaxation algorithm, and compare these with the results using the grid search-based approach. The results of optimization are also compared to those obtained when the robots perform a random 


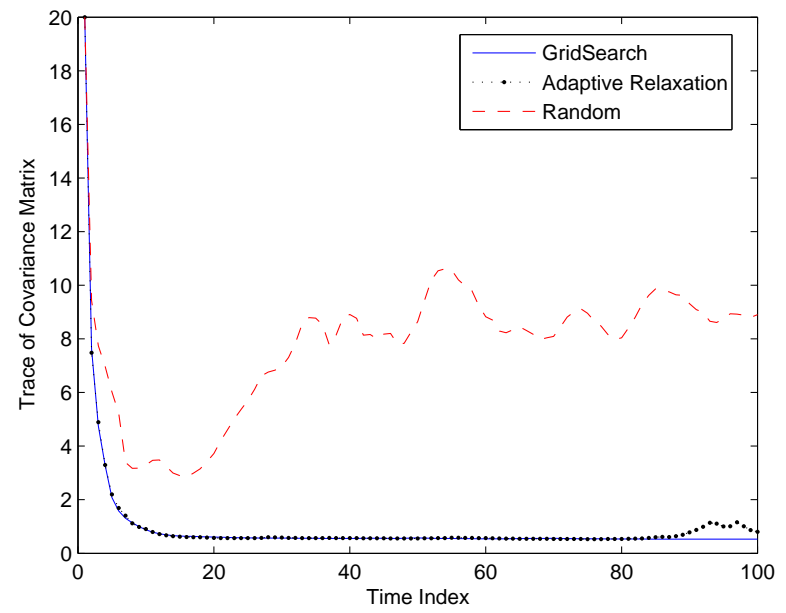

Fig. 3. Trace of the target position covariance matrix. Comparison between the Grid-Search, Adaptive Relaxation, and Random motion strategies.

motion. For our experiments, we adopt a zero-acceleration target motion model:

$$
\ddot{x}=w_{x}, \ddot{y}=w_{y}
$$

and thus the target state is:

$$
X_{T}(k)=\left[x_{T}(k) y_{T}(k) \dot{x}_{T}(k) \dot{y}_{T}(k)\right]^{\mathrm{T}}
$$

The time step is $\delta t=0.1 \mathrm{sec}$, the number of robots is $M=3$, the initial state uncertainty is given by $P_{0 \mid 0}=$ $10 I_{4 \times 4}$, the continuous-time state propagation noise covariance is $Q=10 I_{2 \times 2}$, the robots' maximum speed is $10 \mathrm{~m} / \mathrm{sec}$, the measurement noise covariance is $R=I_{3 \times 3}$, and the number of iterations in the adaptive relaxation algorithm is set to 20 . The relaxation factor used is $\alpha=0.5$. For the grid search method, the grid size is set to $\pi / 200$, which is approximately 1 degree. This is limited by the available computational resources.

For the results shown in Fig.s 3 and 4, the initial state of the target is $X_{T}(k)=\left[\begin{array}{llll}0 & 0 & -8 & 4\end{array}\right]^{\mathrm{T}}$. All robots start from the same position, which is at a distance $15 \mathrm{~m}$ from target. The trajectories of the robots and the target are depicted in Fig. 4. As shown in Fig. 3, the values achieved for the trace of the target-position covariance matrix are very close for the grid search method and the adaptive relaxation algorithm, and they are far better compared to those obtained when the robots move randomly.

\section{CONCLUSIONS}

In this paper, we study the problem of determining optimal trajectories for a team of robots that track a moving target using range-only measurements. The optimality criterion used is the minimization of the trace of the target's position covariance matrix or, equivalently for this case, the minimization of the maximum eigenvalue of this matrix. We have demonstrated that minimization of the trace can be reformulated into minimization of the 2-norm of the sum of a number of vectors in 2-D which have fixed lengths but can change directions within a certain range. Since this problem is non-convex, an adaptive relaxation algorithm is introduced to search for a globally optimal solution. The computational complexity and

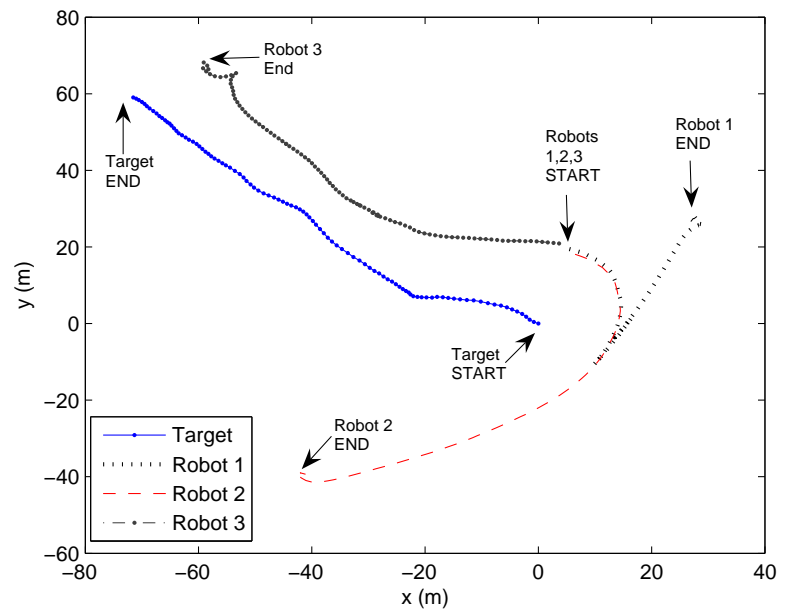

Fig. 4. Trajectories of Target and Robots.

memory requirements of the adaptive relaxation algorithm are significantly lower compared to those of a grid search method (linear vs. exponential in the number of robots). The simulation results show that the adaptive relaxation algorithm will result in almost identical performance to that of a grid search method, and yields far better results when compared to the case when the robots move randomly. In our future work, we will consider the case when the localization of the robots is not perfect, and take into account the uncertainty of their positions. Moreover, it is interesting to solve the problem for the scenario where the robots have bearing-only, or both distance and bearing measurements.

\section{REFERENCES}

[1] L. E. Parker, B. Birch, and C. Reardon, "Indoor target intercept using an acoustic sensor network and dual wavefront path planning."

[2] G. M. Siouris, G. Chen, and J. Wang, "Tracking an incoming ballistic missile using an rxtended interval kalman filter," IEEE Transactions on Aerospace and Electronic Systems, vol. 3, no. 1, pp. 232-340, 1997.

[3] A. Ribeiro, G. B. Giannakis, and S. I. Roumeliotis, "Soi-kf: Distributed kalman filtering with low-cost communications using the sign of innovations," IEEE Transactions on Signal Processing, vol. 54, 2006, (to appear).

[4] R. Vidal, O. Shakernia, H. J. Kim, D. H. Shim, and S. Sastry, "Probabilistic pursuit-evasion games: theory, implementation, and experimental evaluation," IEEE Trans. Robot. Automat., vol. 18, no. 5, pp. 662-669, Oct 2002.

[5] A. I. Mourikis and S. I. Roumeliotis, "Optimal sensing strategies for mobile robot formations: Resource constrained localization," in Proc. Robotics: Science and Systems Conference, Cambridge, MA, June 8-11 2005, pp. 281-288.

[6] R. Barsanti and M. Tummala, "Parameter estimation for target tracking with uncertain sensor positions," in IEEE International Symposium on Circuits and Systems, vol. 2, May. 2001, pp. 257-260.

[7] Y. Bar-Shalom, X. R. Li, and T. Kirubarajan, Estimation with Applications to Tracking and Navigation. Wiley-Interscience, 2001.

[8] H. J. S. Feder, J. J. Leonard, and C. M. Smith, "Adaptive mobile robot navigation and mapping," Int. J. Robotics Research, vol. 18, no. 7, pp. 650-668, July 1999.

[9] V. Christopoulos and S. I. Roumeliotis, "Multirobot trajectory generation for single source explosion parameter estimation," in In Proc. 2005 International Conference on Robotics and Automation, Barcelona, Spain, April 18-22 2005, pp. 2814-2820.

10] N. Trawny and T. Barfoot, "Optimized motion strategies for cooperative localization of mobile robots," in Proceedings of the IEEE International Conference on Robotics and Automation (ICRA), vol. 1, New Orleans, LA, April 26 - May 1 2004, pp. 1027-1032.

[11] A. W. Stroupe and T. Balch, "Value-based action selection for observation with robot teams using probabilistic techniques," Robotics and Autonomous Systems, vol. 50, no. 2, pp. 85-97, 2005.

[12] K. Zhou and S. I. Roumeliotis, "Optimal motion strategies for rangeonly distributed target tracking," University of Minnesota, Tech. Rep., 2006, http://mars.cs.umn.edu/tech_reports/TTSMO.pdf. 